\title{
Correction to: Exploring how medical students learn with the help of a digital presentation: a qualitative study
}

Mary Hyll ${ }^{1,2^{*}}$, Robert Schvarcz ${ }^{1,3}$ and Katri Manninen ${ }^{3}$

\section{Correction to: BMC Med Educ}

https://doi.org/10.1186/s12909-019-1569-z

Following publication of the original article [1], the author reported that Table 3 was given the incorrect heading.

Incorrect heading for Table $\mathbf{3}$ in the original article:

Radiographic examination of $\mathrm{BH}$ (changes in bone height surrounding the implant).

\section{Correct heading for Table 3:}

Overview of the sub-categories, categories, sub-themes and main themes which emerged in the study.

The original article has been corrected.

\section{Author details}

'Department of Medicine Huddinge, Karolinska Institutet, Huddinge, 14186 Stockholm, Sweden. ${ }^{2}$ Department of Infectious Diseases 163, Karolinska University Hospital, Huddinge, 14186 Stockholm, Sweden. ${ }^{3}$ Department of Infectious Diseases 173, Karolinska University Hospital, Huddinge, 14186

Stockholm, Sweden.

Published online: 08 July 2019

\section{Reference}

1. Hyll, et al. Exploring how medical students learn with the help of a digital presentation: a qualitative study. BMC Med Educ. 2019;19:210. https://doi. org/10.1186/s12909-019-1569-z.

\footnotetext{
* Correspondence: mary.hyll@ki.se

'Department of Medicine Huddinge, Karolinska Institutet, Huddinge, 14186 Stockholm, Sweden

2Department of Infectious Diseases 163, Karolinska University Hospital,

Huddinge, 14186 Stockholm, Sweden

Full list of author information is available at the end of the article
} 